\title{
Mortality and Disease Burden of Injuries from 2008 to 2017 in Anhui Province, China
}

\author{
Xiu-Ya Xing, ${ }^{1}$ Peng Wang, ${ }^{2}$ Zhiwei Xu, ${ }^{3}$ Qin He, ${ }^{1}$ Rui Li, ${ }^{1}$ Ye-Ji Chen, ${ }^{1}$ Li-Na Liu, ${ }^{4}$ \\ Yan-Mei Mao, ${ }^{4}$ Chan-Na Zhao, ${ }^{4}$ Yi-Lin Dan, ${ }^{4}$ Qian Wu, ${ }^{4}$ Hai-Feng Pan ${ }^{(D},{ }^{4}$ Zhi-Rong Liu $\left(\mathbb{D},{ }^{1}\right.$ \\ and Wenbiao $\mathrm{Hu}$ (D) $^{3}$ \\ ${ }^{1}$ Department of Chronic Non-communicable Disease Prevention and Control, Anhui Provincial Center for Disease Control \\ and Prevention, Hefei, Anhui 230601, China \\ ${ }^{2}$ Center for Genetic Epidemiology and Genomics, School of Public Health, Medical College of Soochow University, 199 Renai Road, \\ Suzhou, Jiangsu, China \\ ${ }^{3}$ School of Public Health and Social Work, Institute of Health and Biomedical Innovation, Queensland University of Technology, \\ Brisbane, Queensland, Australia \\ ${ }^{4}$ Department of Epidemiology and Biostatistics, School of Public Health, Anhui Medical University, 81 Meishan Road, Hefei, \\ Anhui 230032, China
}

Correspondence should be addressed to Hai-Feng Pan; panhaifeng@ahmu.edu.cn, Zhi-Rong Liu; liuzhirong66@126.com, and Wenbiao Hu; w2.hu@qut.edu.au

Received 3 September 2019; Accepted 15 February 2020; Published 22 April 2020

Academic Editor: Pasquale De Bonis

Copyright (c) 2020 Xiu-Ya Xing et al. This is an open access article distributed under the Creative Commons Attribution License, which permits unrestricted use, distribution, and reproduction in any medium, provided the original work is properly cited.

Objective. To investigate the temporal trends in mortality and disease burden of injuries in Anhui province from 2008 to 2017, so as to provide reference for injury control and prevention. Methods. Data of mortality were collected from 9 national surveillance points in Anhui province during 2008-2017 in the Information System for Death Cause Register and Management. The surveillance data were analyzed by using crude mortality, standardized mortality rate (SMR), potential year of life lost (PYLL), PYLL rate (PYLLR), and average of year life lost (AYLL). Results. There were a total of 44855 people died from injury, accounted for $9.44 \%$ of the all-cause mortality, ranked as the fifth leading cause of deaths in the whole population, and denoted the first leading cause of deaths in the 0-44 year's group. The leading causes of injury deaths were road traffic accidents, suicide, accidental falls, drowning, and poisoning. Road traffic accidents was the primary cause of injury deaths among the male population, while suicide was the dominate cause of injury deaths among the female population. Drowning, traffic accidents, and suicide accounted for the most injury deaths among the population aged 0-14 years, 15-64 years, and above 60 years, respectively. The road traffic accidents accounted for the largest proportion of injury PYLL and PYLLR, and drowning caused the highest AYLL among injury deaths. Conclusion. In Anhui province, road traffic accidents, suicide, accidental falls, drowning, and poisoning were the top five causes of injury deaths that harm the health of local residents; corresponding injury prevention strategies should be formulated.

\section{Introduction}

Injury, communicable diseases, and chronic noncommunicable diseases are listed as the three major burdens of disease worldwide [1]. Road traffic accidents, suicide, accidental falls, drowning, and accidental poisoning are the common types of injury [2-4]. It has been estimated that there are more than 5 million people died from injuries each year worldwide $[5,6]$. Although the majority of deaths caused by injuries (over 90\%) occurred in low- and middleincome countries, various types of injuries collectively have become an increasing public health issue that threaten the health of people and rank as the fifth cause of deaths in China [2, 7-9]. From 1995 to 2008, it has been reported that there were about 0.70 to 0.75 million people who died from injuries each year, accounted for $9 \%$ of all-cause 
mortality in China $[10,11]$. In addition to the serious harms of injury on people's life and health, the consequently heavy financial burden cannot be ignored [12], as the total economic burden caused by injury is more than 379.231 billion Yuan in China [13].

The network report system that recorded the mortality data has been established in China since 2008. Anhui, a province located in the eastern region of China, is the $22^{\text {nd }}$ largest Chinese province based on area, the $8^{\text {th }}$ most populous province, and the $12^{\text {th }}$ most densely populated region in the whole country. In Anhui province, the mortality statistics is often generated from cross-sectional population based surveys, and the related trend analysis has not been carried out yet.

In the present study, in order to derive a precise evaluation on the status regarding injury mortality and provide reference for formulating injury control and prevention strategies, we investigated the temporal trends in mortality and disease burden of injury in Anhui province from 2008 to 2017. In addition, we further performed a detailed analysis of the leading causes of injury-related mortality.

\section{Methods}

2.1. Data Sources. In the present study, data of mortality were obtained from the Information System for Death Cause Register and Management covering 9 national surveillance points in Anhui province from year of 2008 to 2017. The nine national surveillance points included Juchao district of Hefei, Yushan district of Maanshan, Tianchang city of Chuzhou, Jing county of Xuancheng, Mengcheng county of Bozhou, Shouxian county of Luan, Lingbi county of Suzhou, Yingdong district of Fuyang, and Yongqiao district of Suzhou, where the location of mentioned the 9 surveillance points was evenly distributed within Anhui province. The nine surveillance points have a good quality control, the mortality rate is above $5.5 \%$, and the correct encoding rate is $98.00 \%$. The population demographic data were retrieved from the Resident Population Information System of Chinese Center for Disease Control and Prevention (China CDC).

This study was approved by the ethical committee of Anhui Provincial Center for Disease Control and Prevention. Data obtained from the reporting system did not have any personal identifiers; privacy and confidentiality of all information were maintained.

2.2. Classification of Injury Death. The classification of injury death was based on the criteria of the International Statistical Classification of Diseases and Related Health Problems (WHO), Tenth Revision (ICD-10), edition 2 [14, 15].
Damage (V01-Y89) includes accidental injury (V01-X59, Y40-Y86, Y88, and Y89) and intentional injury (X60-Y09, Y35-Y36, Y87.0, and Y87.1); accidental injury represents road traffic accidents (V01-V04, V06, V09-V80, V87, V89, and V99), accidental poisoning (X40-X49), accidental falls (W00-W19), fire disaster (X00-X09), drowning (W65-W74), and other accidental injuries (V05, V07-V08, V81-V86, V88, V90-V98, W20-W64, W75-W99, X10-X39, X50-X59, Y40-Y86, Y88, and Y89); intentional injury includes suicide and its sequelae (X60-X84, Y87.0), homicide and its sequelae (X8-Y09, Y87.1), war (Y36), and other intentional injuries (Y35).

2.3. Statistical Analysis. Data arrangement and analysis were implemented by Microsoft Excel and SPSS 23.0 statistical software. The epidemiological characteristics of injury death and trends were analyzed and assessed by crude mortality rate, standardized mortality rate (SMR), composition ratio, rank order of cause of death, and the annual percentage change in mortality rate (APC). The comparison of mortality rate between two groups was performed using $Z$ test, with $p<0.05$ for statistical significance.

To emphasize the influence of injury caused early death on the life reducing, the following terms were applied to evaluate the disease burden of injury, including potential years of life lost (PYLL), PYLL rate (PYLLR), and average years of life lost (AYLL). The SMR was calculated according to the $6^{\text {th }}$ national population census of China. According to the maximum expected value method, the life expectancy of 70 years was regarded as the assumed life expectancy to calculate the relevant indicators of life reduction. The age range of "premature death" was from 1 to 69 years old. The calculation method was as follows:

$$
\begin{aligned}
\text { Mortality rate }= & \text { the number of deaths/total population } \\
& \times 100000 / 100 \text { thousand, } \\
\text { APC }= & \left(10^{\mathrm{a}}-1\right) \times 100 \%,
\end{aligned}
$$

where the logarithm of mortality in each year is the dependent variable and year is the independent variable; the fitting line was $Y=b+a x$, the value of $a$ was obtained, $Y=\log$ (mortality rate), and $x=$ year. Then the hypothesis test is carried out on APC to check whether APC is an accidental change.

PYLL $=\sum$ (life expectancy - median for each age group $0.5) \times$ number of deaths in each age group.

$\mathrm{PYLLR}=\mathrm{PYLL} / n \times 1,000 \%$ ( $n$ is the total population age from 1 to 69 years in the target population $),$

AYPLL $=$ YPLL/ $d(d$ is the total number of deaths from a cause of death in the target population aged from 1 to 69 years $)$. 


\section{Results}

3.1. Injury Deaths Profile. From 2008 to 2017, a total of $74,225,139$ people $(37,509,558$ males and $36,715,581$ females) were recruited within 9 national surveillance points in Anhui province, and 44855 people (30213 males and 14642 females) died from injuries, with an average crude mortality rate of 60.43/100000 (SMR: 59.50/100000), accounting for $9.44 \%$ of the all-cause mortality (44855/475177). In terms of gender distribution of injury mortality, the average male crude injury mortality rate was $80.55 / 100000$ (SMR: $82.53 / 100000$ ), accounting for $10.90 \%$ of the all-cause mortality in males (30213/277073), and the average female crude injury mortality rate was 39.88/100000 (SMR: 36.98/100000), accounting for $7.39 \%$ of the all-cause mortality in females (14642/198104). The difference of injury SMR between the male and female groups was statistically significant $(Z=80.13, p<0.001)$, with an increased injury SMR in male than in female groups. The injury death ranked $5^{\text {th }}$ of causes of death after cerebrovascular disease, malignant tumor, and heart and respiratory diseases, and denoted the first cause of death in the group of age 0-44 years.

3.2. Cause of Injury Deaths. In Anhui province, from 2008 to 2017, the top five causes of injury deaths were road traffic accidents, suicide, accidental falls, drowning, and accidental poisoning, accounting for $87.26 \%$ cause of injury deaths. The first leading cause of injury deaths for males was road traffic accidents, followed by suicide; while for females, suicide was the leading mechanism of injury deaths, followed by road traffic accidents; the other rank order of cause of injury deaths in both males and females was consistent (Table 1).

The rank order of cause of injury deaths in different age groups showed significant differences, among which road traffic accidents, drowning, and suicide were the top three causes of injury deaths among each age subgroup. Drowning was the leading cause of injury deaths in the age group of $0-14$ years old and contributed for $41.78 \%$ of the cause of injury deaths; moreover, road traffic accidents were the first leading cause of injury deaths in the age group of 15-44 and $45-64$ years old $(49.07 \%$ and $43.29 \%)$. Suicide was the first leading cause of injury deaths in the age group above 65 years old and accounted for $36.62 \%$ of the cause of injury deaths in this age group. In addition, road traffic accidents ranked as the second cause of injury deaths in the age group of 0-14 and over 65 years old $(25.54 \%$ and $23.57 \%)$. Furthermore, suicide was the second rank cause of injury deaths in the age group of 15-44 and 45-64 years old (17.12\% and $23.47 \%$ ). Accidental fall-caused death in the age group 65 and older accounted for $52.93 \%$ of the total number of accidental fall death in all age groups (Table 1).

\subsection{Trend of Injury Mortality Rates in Different Genders.} During the past 10 years (2008-2017), the injury mortality rate of residents in Anhui province declined by $4.72 \%$ each year. The decrease of SMR between male and female groups was statistically significant $(p<0.001)$, with the SMR of $4.28 \%$ in males and $5.81 \%$ in females. When the SMR is compared between different genders in the same year, there was a higher SMR in male group than those of female group (all $p<0.05$ ) (Table 2).

3.4. Trends in Injury Mortality Rates in Different Age Groups. From 2008 to 2017, the trends of age-specific injury mortality rate showed a steady decline (Figure 1). The age group of 15 to 44 years old had the greatest decreasing rate of APC, and the age group of above 65 years old had the lowest decreasing APC, as compared with other age groups (Figure 2).

3.5. Trends in Major Injury Mortality Rates. From 2008 to 2017, the trend of the top five injury mortality rates was different, among which the SMR of road traffic accidents, suicides, and accidental falls displayed a significant downward trend (all $p<0.05$ ), with the APC of $4.19 \%, 8.16 \%$, and $2.66 \%$, respectively. Furthermore, the road traffic accidents SMR peaked in 2011 and then showed a downward trend, with the largest decline in 2013 to 2014. However, the trend of SMR in drowning and accidental poisoning was not significant (Table 3 ).

3.6. The Evaluation of Disease Burden in Injury Deaths. During the past 10 years, the PYLL was 830147 person-years attributed to injury and accounted for $33.89 \%$ of all-cause mortality (2449302 person-years); the PYLLR was $12.15 \%$ and AYLL was 26.33 years, and the results indicated that injury ranked $1^{\text {st }}$ of all-cause mortality. When analyzing the gender-specific disease burden, the PYLLR was higher in male group than in female group $(17.41 \%$ vs. $6.74 \%)$; although the difference regarding AYLL was only 0.70 years between male and female groups, the injury PYLL in male was 2.65 times higher than in female (602722 person-years vs. 227425 person-years). The top five causes of injury deaths contributed in PYLL were road traffic accidents, suicides, drowning, accidental falls, and accidental poisoning. While, the top five causes of injury deaths in AYLL were drowning, homicide, road traffic accidents, fire disaster, and accidental poisoning (Table 4).

\section{Discussion}

In comparison to the frequency on occurrence of injury, injury deaths are only the "tip of the iceberg." It has been estimated that the injury deaths and occurrence ratio is about $1: 100$ in China. Injury is characterized as high incidence rate, high disability rate, and high mortality rate, as well as an often-neglected common health issue.

In our study, we revealed that, from 2008 to 2017, the average crude injury mortality rate of residents was 60.43/100000 (SMR: 59.50/100000) in Anhui province and ranked $5^{\text {th }}$ of all-cause mortality. As compared with other provinces, there was an increased SMR in Anhui province (59.50/100000) than in Chongqing (55.57/100000), Guangxi Zhuang autonomous region (46.45/100000), Guangdong province (43.11/100000), Wuxi city (38.62/100000), and nationwide (44.98/100000) [16-20]; however, the SMR of Anhui province was similar to that in Taizhou (60.65/100000) [21]. In Anhui province, injury mortality rates displayed a descending trend year by year from 2008 


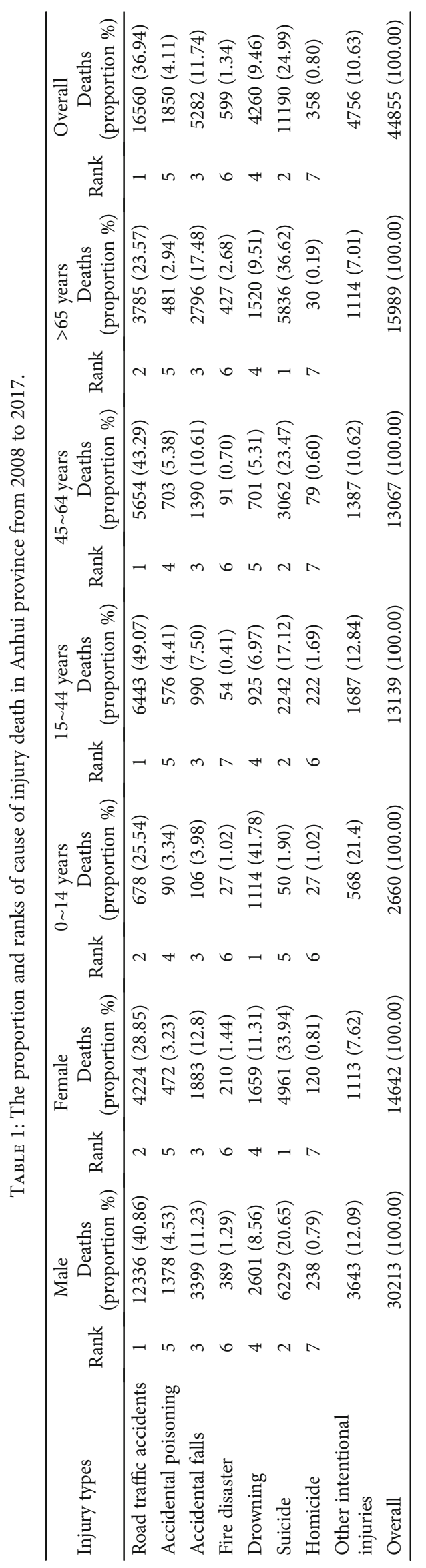


TABLE 2: The trends of gender-specific injury mortality rates (1/100000) in Anhui province from 2008 to 2017.

\begin{tabular}{|c|c|c|c|c|c|c|c|c|c|}
\hline \multirow{2}{*}{ Year } & \multicolumn{3}{|c|}{ Male } & \multicolumn{3}{|c|}{ Female } & \multicolumn{3}{|c|}{ Overall } \\
\hline & Deaths & Crude mortality rate & SMR & Deaths & Crude mortality rate & SMR & Deaths & Crude mortality rate & SMR \\
\hline 2008 & 3286 & 83.65 & $97.82^{\mathrm{a}}$ & 1659 & 44.69 & 48.32 & 4945 & 64.72 & 72.91 \\
\hline 2009 & 3230 & 81.81 & $94.94^{\mathrm{a}}$ & 1672 & 44.84 & 47.92 & 4902 & 63.85 & 70.86 \\
\hline 2010 & 3268 & 82.51 & $93.43^{\mathrm{a}}$ & 1531 & 40.86 & 43.16 & 4799 & 62.26 & 68.22 \\
\hline 2011 & 3251 & 89.22 & $98.19^{\mathrm{a}}$ & 1578 & 44.30 & 43.34 & 4829 & 67.01 & 69.80 \\
\hline 2012 & 3289 & 90.88 & $90.32^{\mathrm{a}}$ & 1550 & 43.00 & 38.38 & 4839 & 66.99 & 63.90 \\
\hline 2013 & 3138 & 86.72 & $88.43^{\mathrm{a}}$ & 1443 & 40.04 & 37.19 & 4581 & 63.42 & 62.52 \\
\hline 2014 & 2729 & 74.53 & $75.15^{\mathrm{a}}$ & 1281 & 35.47 & 32.25 & 4010 & 55.13 & 53.52 \\
\hline 2015 & 2681 & 74.13 & $73.64^{\mathrm{a}}$ & 1273 & 34.27 & 31.04 & 3954 & 53.93 & 51.84 \\
\hline 2016 & 2740 & 73.66 & $71.10^{\mathrm{a}}$ & 1352 & 36.27 & 30.69 & 4092 & 54.94 & 50.62 \\
\hline 2017 & 2601 & 68.58 & $67.83^{\mathrm{a}}$ & 1303 & 35.19 & 30.86 & 3904 & 52.08 & 49.17 \\
\hline Overall & 30213 & 80.55 & 82.53 & 14642 & 39.88 & 36.98 & 44855 & 60.43 & 59.50 \\
\hline $\operatorname{APC}(\%)$ & & & -4.28 & & & -5.81 & & & -4.72 \\
\hline$t$ & & & -7.71 & & & -9.42 & & & -10.68 \\
\hline$p$ & & & $<0.001$ & & & $<0.001$ & & & $<0.001$ \\
\hline
\end{tabular}

${ }^{a}$ Compared with the female in the same year $(p<0.05)$. APC: annual percentage change in mortality rate; SMR: standardized mortality rate.

\begin{tabular}{lcccc}
\hline \multicolumn{5}{c}{ Age specific injury mortality rate $(1 / 100,000)$} \\
Year & $0-14$ years & $15-44$ years & $45-64$ years & above 65 years \\
\hline 2008 & 20.93 & 43.15 & 87.64 & 253.86 \\
2009 & 23.1 & 43.08 & 80.63 & 248.61 \\
2010 & 23.36 & 38.58 & 88.94 & 232.15 \\
2011 & 19.34 & 41.05 & 97.52 & 219.65 \\
2012 & 23.85 & 42.27 & 83.01 & 211.36 \\
2013 & 20.22 & 42.66 & 72.18 & 204.83 \\
2014 & 17.63 & 32.84 & 61.63 & 199.1 \\
2015 & 17.23 & 30.73 & 60.91 & 191.06 \\
2016 & 15.49 & 27.41 & 65.95 & 185.63 \\
2017 & 12.48 & 24.42 & 63 & 192.98 \\
\hline
\end{tabular}

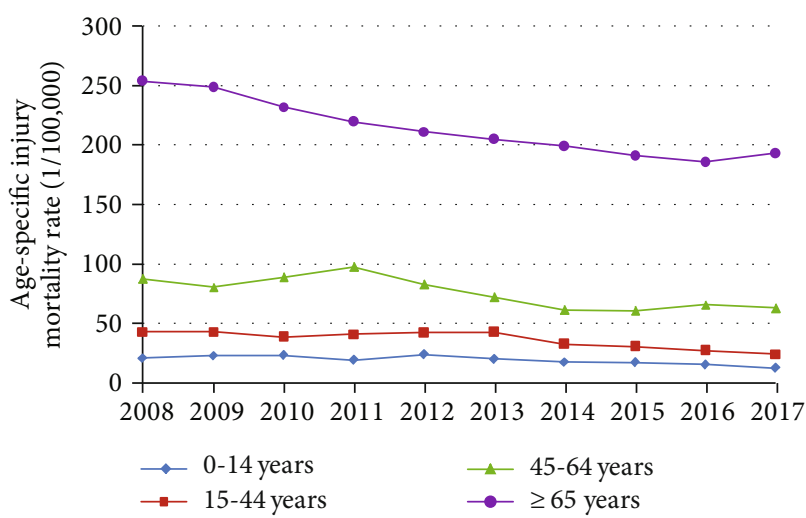

FIgURE 1: The trends of age-specific injury mortality rates (1/100000) in Anhui province from 2008 to 2017.

\begin{tabular}{lc}
\hline & $\begin{array}{c}\text { Annual percentage change in } \\
\text { mortality rate }\end{array}$ \\
\hline 0-14 years & 5.55 \\
15-44 years & 5.93 \\
45-64 years & 4.609 \\
$\geq 65$ years & 3.46 \\
\hline
\end{tabular}

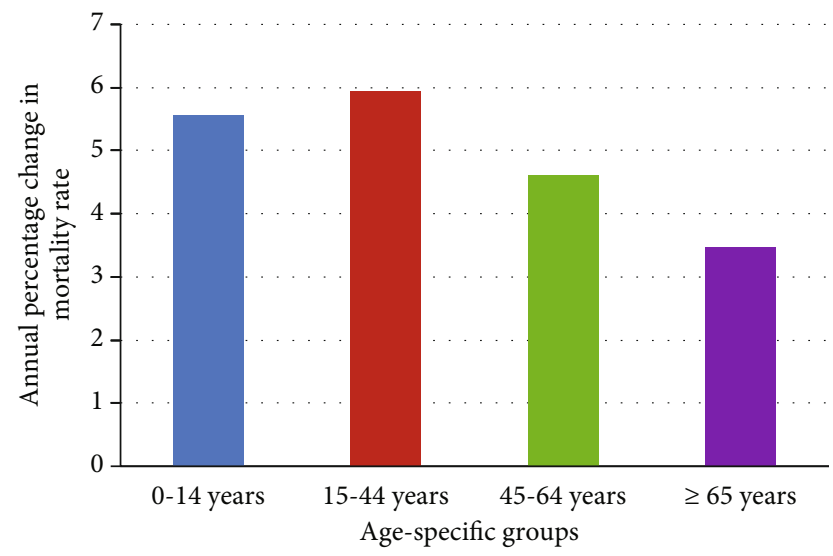

FIGURE 2: Annual percentage changes in mortality rate among different age groups from 2008 to 2017. 


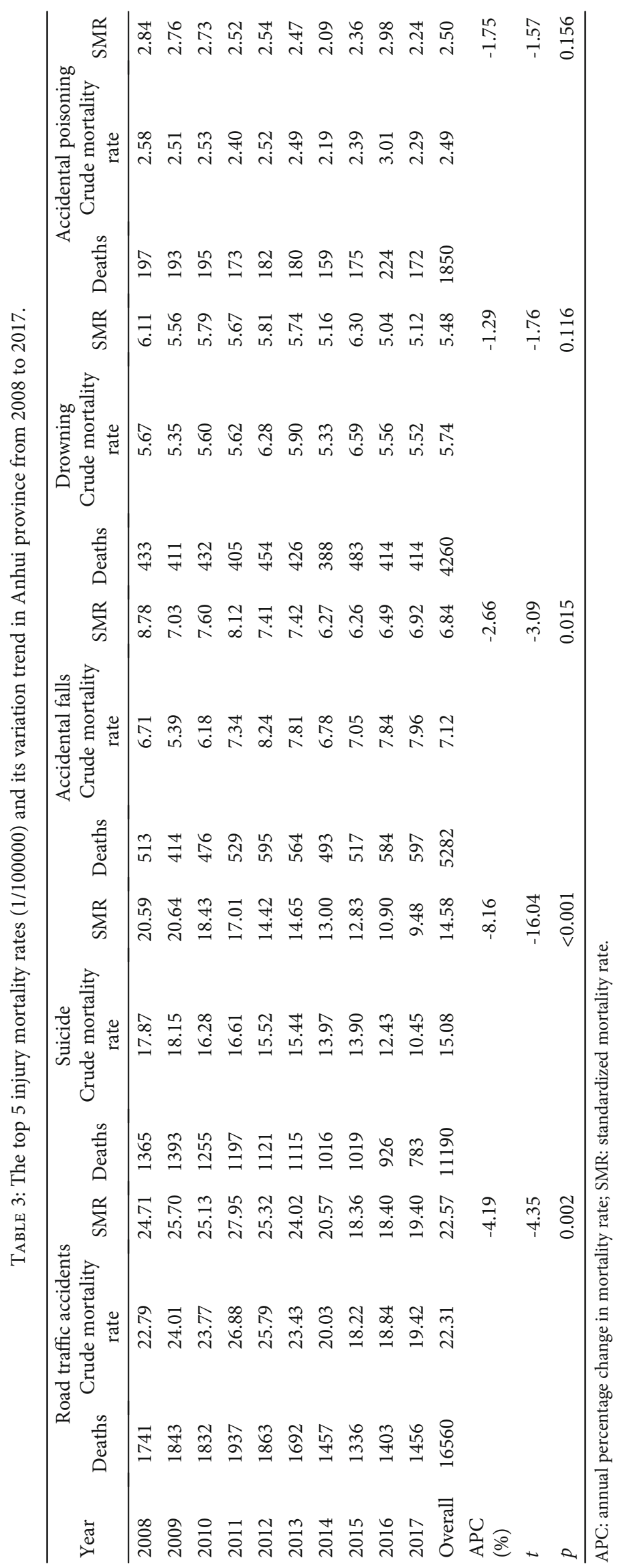




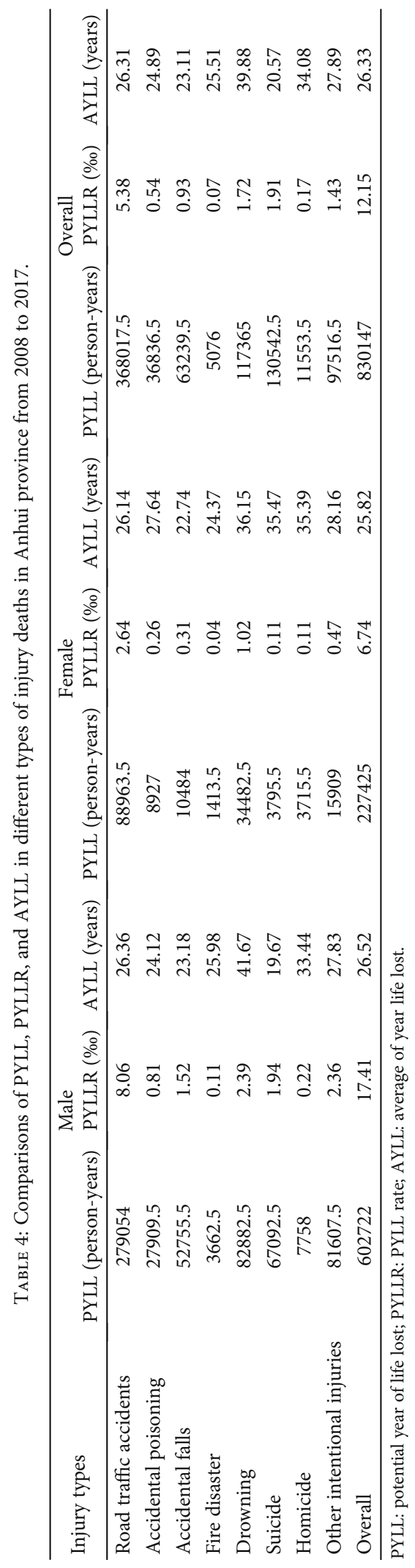


to 2017, and this trend was consistent with the national and some regional reports. The top three leading causes of injury deaths were road traffic accidents, suicide, and accidental falls; this mortality spectrum of injuries in Anhui province was also similar to the national situation.

The SMR of injury mortality were higher in male than in female groups each year, as well as a 2.65 times increase of PYLL and 2.58 times PYLLR in male than in female groups; this result was consistent with previous studies [20-23], suggesting the higher injury burden in male. In fact, PYLL, PYLLR, and other indicators focused on the description of premature death, emphasizing the loss of life caused by premature death $[22,24]$. The increased SMR of injury deaths, PYLL, and PYLLR in male may be related to the fact that they have to take much more social responsibilities and usually work on physical labor that made them in higher risktaking behaviors than female $[17,25,26]$.

Road traffic accidents were the first cause of injury deaths and the most important cause of life loss in Anhui province. The PYLL of road traffic accidents was 368017.5 years, accounting for $44.33 \%$ of all PYLL caused by injury. It may be explained by a rapid increase in the number of vehicles over the past few years, with inevitable surges in accidents and the traffic jam, as well as the lower safety awareness $[27,28]$. Furthermore, the SMR of road traffic accidents present two turning points: one is the downtrend year by year after the peak in 2011 and the other is that there was the largest annual descend range from 2013 to 2014. One important underlying reason for this change of trends may be explained by the promulgation of "China's newly-amended Road Traffic Safety Law" which took effect on May 2011. The newly implemented amendment law relatively imposed new stiff penalties, especially criminal charges on persons with a drunk driving. In all, the improvement of public awareness on traffic safety and road conditions and promulgation of relevant laws and regulations are effective measures to reduce traffic fatal-accident rate.

Suicide was the second cause of injury deaths in Anhui province and the first cause of injury death in women, with a higher AYLL of suicide in women than in men; this is closely associated with the special psychological and physiological characteristics of women. Studies have revealed that, as compared with men, women have a higher neuroticism score and are inclined to take negative coping strategies; thus, they are easily to have a suicidal tendency $[18,29]$. Moreover, we also found that suicide ranked $1^{\text {st }}$ of injury deaths in age above 65 years old, which was similar to the results of Shandong province, Guangxi province, and Hunan province in China [22, 30]. Several studies have demonstrated that suicide has a relationship with psychological status, family conflicts, and healthy condition. The elderly people, as a vulnerable group, gained fewer social supports and were more likely to have a suicidal behavior because of family conflicts and diseases. In recent years, the suicide rate in Chinese rural elderly people has been increasing [31]; therefore, the government health care/service and family psychological health education should be allocated to improve the psychological status on both female and elder people, to reduce the incidence/mortality rate of suicide.
Accidental falls were the third cause of injury deaths in all age groups, among which the age group above 65 years old showed a higher incidence of accidental fall deaths than the other age groups, accounting for $17.48 \%$ of accidental fall deaths. The study performed by Shao et al. also suggested that elder people are prone to accidental falls [32]. Anhui is one of the main sources of rural labor service export. The phenomenon of left-behind elderly people in rural areas has become a common social issue, such that many elderly people in rural areas have to do housework, do farm work, and help their children babysit the new born, but the lack of family care on elderly people makes them easily fall down. Therefore, more attention and care should be paid for the elder people to prevent the possible accidental falls and improve their quality of life.

Drowning represented the leading cause of injury deaths in children age group of $0-14$ years and is the first rank cause of injury deaths in the whole population with premature death. In recent years, incidence of children drowning has been reported frequently $[33,34]$. The risk factors that contributed to the child drowning included children personality characteristics, family factors (inadequate parental supervision and control), and environmental factors (shortness of life-saving equipment and safe guards) [33, 35]. Children drowning cognitive education should be strengthened to enhance their safety awareness; first-aid knowledge and skills for drowning should be provided to improve children's capability in facing emergent situations [36].

There are some limitations that have to be taken into account. First, the incomplete data of Information System for Death Cause Register and Management may underestimate the true injury mortality. Second is the lack of information on potential influencing factors, such as socio-economic status, family background, or detailed conditions; thus, the association of injury deaths with potential influencing factors could not be fully understood.

Injury is one of the main causes of deaths in the residents of Anhui province with age and gender heterogeneity. Traffic accidents, suicide, falls, drowning, and poisoning were the top five causes of deaths and should be targeted for injury prevention activity. Moreover, it is critically pivotal to improve policies and programs that can deliver effective measures in the high-risk populations and areas.

\section{Data Availability}

No additional data are available.

\section{Ethical Approval}

This study was based on the disease surveillance data that was obtained from the reporting system without any personal identifiers; privacy and confidentiality of all information were maintained.

\section{Conflicts of Interest}

The authors declared that they have no conflicts of interests. 


\section{Authors' Contributions}

Xiu-Ya Xing and Peng Wang contributed equally to this work and should be considered cofirst authors.

\section{Acknowledgments}

We would like to thank our colleagues at Anhui Provincial Centre for Disease Control and Prevention for sharing their data for our analysis.

\section{References}

[1] GBD 2017 Disease and Injury Incidence and Prevalence Collaborators, "Global, regional, and national incidence, prevalence, and years lived with disability for 354 diseases and injuries for 195 countries and territories, 1990-2017: a systematic analysis for the Global Burden of Disease Study 2017," The Lancet, vol. 392, no. 10159, pp. 1789-1858, 2018.

[2] L. Zhang, Z. Li, X. Li et al., "Study on the trend and disease burden of injury deaths in Chinese population, 2004-2010," PLoS One, vol. 9, no. 1, article e85319, 2014.

[3] P. Ning, X. Cheng, L. Zhang, W. Zhang, and G. Hu, "Injury mortality in China, from 1990 to 2010," Zhonghua Liu Xing Bing Xue Za Zhi, vol. 36, no. 12, pp. 1387-1390, 2015.

[4] L. Wang, Y. Liu, S. Liu et al., "Status injury burden in 1990 and 2010 for Chinese people," Zhonghua Yu Fang Yi Xue Za Zhi, vol. 49, no. 4, pp. 321-326, 2015.

[5] GBD 2015 DALYs and HALE Collaborators, "Global, regional, and national disability-adjusted life-years (DALYs) for 315 diseases and injuries and healthy life expectancy (HALE), 1990-2015: a systematic analysis for the Global Burden of Disease Study 2015," The Lancet, vol. 388, no. 10053, pp. 1603-1658, 2016.

[6] GBD 2015 Mortality and Causes of Death Collaborators, "Global, regional, and national life expectancy, all-cause mortality, and cause-specific mortality for 249 causes of death, 1980-2015: a systematic analysis for the Global Burden of Disease Study 2015," The Lancet, vol. 388, no. 10053, pp. 1459-1544, 2016.

[7] J. Liang, X. Li, C. Kang et al., "Maternal mortality ratios in 2852 Chinese counties, 1996-2015, and achievement of Millennium Development Goal 5 in China: a subnational analysis of the Global Burden of Disease Study 2016," The Lancet, vol. 393, no. 10168, pp. 241-252, 2019.

[8] A. Chandran, A. A. Hyder, and C. Peek-Asa, "The global burden of unintentional injuries and an agenda for progress," Epidemiologic Reviews, vol. 32, no. 1, pp. 110-120, 2010.

[9] J. Jagnoor, S. Prinja, H. Nguyen, B. J. Gabbe, M. Peden, and R. Q. Ivers, "Mortality and health-related quality of life following injuries and associated factors: a cohort study in Chandigarh, North India," Injury Prevention, vol. 4, article injuryprev-2019-043143, 2019.

[10] S. Y. Wang, "Epidemiological features and research progress of injury in China," Chinese Journal of Epidemiology, vol. 32, no. 7, pp. 637-642, 2011.

[11] S. Y. Wang, Y. H. Li, G. B. Chi et al., "Injury-related fatalities in China: an under-recognised public-health problem," The Lancet, vol. 372, no. 9651, pp. 1765-1773, 2008.

[12] J. Yang, L. Feng, Y. Zheng, and H. Yu, "Estimation on the indirect economic burden of disease-related premature deaths in
China, 2012," Zhonghua liuxingbingxue zazhi, vol. 35, no. 11, pp. 1256-1262, 2014.

[13] Y. M. Zou and Y. T. Hao, "Analysis on years of life lost and economic burden caused by injury in China," Chinese Journal of Disease Control and Prevention, vol. 20, no. 5, pp. 495-499, 2016.

[14] A. M. Minino, R. N. Anderson, L. A. Fingerhut, M. A. Boudreault, and M. Warner, "Deaths: injuries, 2002," National Vital Statistics Reports, vol. 54, no. 10, pp. 1-124, 2006.

[15] I. R. . H. Rockett, M. D. Regier, N. D. Kapusta et al., "Leading causes of unintentional and intentional injury mortality: United States, 2000-2009," American Journal of Public Health, vol. 102, no. 11, pp. e84-e92, 2012.

[16] C. R. Chen, D. K. Zheng, C. Y. Yan, C. Luo, and J. D. Wang, "Reasons for residents on injury death in Wanzhou district of Chongqing: 2005 - 2014," Modern Preventive Medicine, vol. 43, no. 5, pp. 860-863, 2016.

[17] X. Jie, T. N. Feng, S. Ma et al., "Analysis of epidemiological trend and disease burden on injury death in China from 2004 - 2010," Chinese Journal of Hygiene Rescue, vol. 1, no. 4, pp. 294-297, 2015.

[18] Q. L. Qin, W. Mao, Y. M. Teng, J. J. Xu, and J. F. Cai, "Analysis on trend and disease burden of injury deaths in Guangxi Zhuang auonomos region in 2014," Chinese Journal of Disease Control and Prevention, vol. 20, no. 9, pp. 918925, 2016.

[19] J. B. Yang, "Analysis on death condition and disease burden of injury in Wuxi city from 2001 to 2015," Modern Preventive Medicine, vol. 44, no. 4, pp. 660-664, 2017.

[20] R. Meng, X. Xu, Y. Xu et al., "Epidemiological characteristics of injury mortality in Guangdong Province, China, 2015," BMC Public Health, vol. 19, no. 1, p. 142, 2019.

[21] L. Y. Wang, D. J. Qiao, L. L. Zhao et al., "Analysis of epidemiological trend of injury death in Taizhou from 2010 to 2014," Chinese Journal of Prevention and Control of Chronic Diseases, vol. 10, no. 24, pp. 794-796, 2016.

[22] Z. T. Fu, Y. Y. Wang, X. L. Guo, B. Y. Wu, and Z. X. Wang, "Status of injury death and potential years of life lost in Shandong province in 2012," Chinese Journal of Health Statistics, vol. 34, no. 2, pp. 236-239, 2017.

[23] F. Fei, H. Liu, S. I Leuba et al., "Suicide rates in Zhejiang Province, China, from 2006 to 2016: a population-based study," Journal of Epidemiology and Community Health, vol. 73, no. 8, pp. 745-749, 2019.

[24] Centers for Disease Control, "Premature mortality in the United States: public health issues in the use of years of potential life lost," Morbidity and Mortality Weekly Report, vol. 35, no. 2, pp. 1S-11S, 1986.

[25] X. Y. Zhang, G. D. Zhao, B. Hou, and X. T. Du, "Analysis on injury death of residents of Xian: 2004 - 2013," Chinese Journal of Health Statistics, vol. 32, no. 3, pp. 486-488, 2015.

[26] L. Qin and Y. Liu, "Analysis of characteristics of injury death among urban residents in Dandong from 2006-2011," Chinese Journal of Health Statistics, vol. 31, no. 6, pp. 1032-1034, 2014.

[27] X. Y. Xing, W. Xu, Y. J. Chen, Z. R. Liu, Z. P. Bian, and Y. S. Liu, "A roadside observation study of unsafe riding acts among electric bicycle riders in a city of Anhui province," Chinese Journal of Disease Control and Prevention, vol. 21, no. 9, pp. 943-946, 2017. 
[28] C. H. Zhu, X. Gong, C. L. Li, Y. H. Zhu, and H. L. Lv, "Study on influcing factors of injury death and potential years of life lost in a district of Wuhan during 2009 and 2013," Medicine and Society, vol. 29, no. 7, pp. 20-26, 2016.

[29] J. Zhou, "Analysis of death condition of accidental injury in Hailing district from 2011 to 2015," Preventive Medicine and Public Health, vol. 26, no. 13, pp. 50-52, 2016.

[30] Y. X. Zhang, H. B. Ye, L. X. Sun, Y. X. Pan, and T. Liu, “Analysis of causes and trend of injury death in disease monitoring regions of Guizhou in 2008-2012," Modern Preventive Medicine, vol. 41, pp. 1522-1529, 2014.

[31] W. L. Wang, "Suicide of the elderly population in China," Population \& Development, vol. 19, no. 1, pp. 83-89, 2013.

[32] Y. Q. Shao, Z. H. Chen, J. F. Li, L. H. Fan, G. X. Shao, and R. Y. Chen, "Epidemiological characteristics and disease burden of injury death in Wenzhou 2010-2014," Disease Surveilliance, vol. 31, no. 8, pp. 697-702, 2016.

[33] L. Xiang, K. Wang, L. Miao et al., "Injury-related mortality among children younger than 5 years in China during 20092016: an analysis from national surveillance system," Injury Prevention, vol. 25, no. 1, pp. 60-66, 2019.

[34] Y. Wu, D. C. Schwebel, and G. Hu, "Disparities in unintentional occupational injury mortality between high-income countries and low- and middle-income countries: 1990(-) 2016," International Journal of Environmental Research and Public Health, vol. 15, no. 10, p. 2296, 2018.

[35] X. Deng, Y. Jin, P. P. Ye, L. H. Wang, and L. L. Duan, "Disease burden on drowning in the Chinese population, in 1990 and 2013," Zhonghua liuxingbingxue zazhi, vol. 38, no. 10, pp. 1308-1314, 2017.

[36] Q. L. Wu, J. Wang, R. Xu, and Q. H. Xu, "Analysis of no-intentional injury deaths among under five in Suzhou during 2009-2014," Chinese Journal of Women and Child Health, vol. 6, no. 4, pp. 9-12, 2015. 